\title{
Effects of semantic relatedness on recall of stimuli preceding emotional oddballs
}

\author{
RYAN M. SMITH ${ }^{1}$ AND DAVID Q. BEVERSDORF ${ }^{1,2}$ \\ ${ }^{1}$ Neuroscience Graduate Studies Program, The Ohio State University, Columbus, Ohio \\ ${ }^{2}$ Departments of Radiology, Neurology, Psychology, and The Thompson Center, University of Missouri, Columbia, Missouri \\ (Received April 23, 2007; Final Revision March 27, 2008; Accepted March 28, 2008)
}

\begin{abstract}
Semantic and episodic memory networks function as highly interconnected systems, both relying on the hippocampal/medial temporal lobe complex (HC/MTL). Episodic memory encoding triggers the retrieval of semantic information, serving to incorporate contextual relationships between the newly acquired memory and existing semantic representations. While emotional material augments episodic memory encoding at the time of stimulus presentation, interactions between emotion and semantic memory that contribute to subsequent episodic recall are not well understood. Using a modified oddball task, we examined the modulatory effects of negative emotion on semantic interactions with episodic memory by measuring the free-recall of serially presented neutral or negative words varying in semantic relatedness. We found increased free-recall for words related to and preceding emotionally negative oddballs, suggesting that negative emotion can indirectly facilitate episodic free-recall by enhancing semantic contributions during encoding. Our findings demonstrate the ability of emotion and semantic memory to interact to mutually enhance free-recall. (JINS, 2008, 14, 620-628.)
\end{abstract}

Keywords: Association learning, Memory, Short-term, Emotional stress, von Restorff, Oddball

\section{INTRODUCTION}

Declarative memories are categorized as episodic, referring to an autonoetic conscious recollection of a specific experience, or semantic, referring to concept-based knowledgeof-the-world in which the "self" is not present (Tulving, 1983, 2002; Tulving \& Markowitsch, 1998). Neuroimaging evidence supports the existence of separate but highly interconnected semantic and episodic memory systems during encoding, consolidation, and retrieval (Burianova \& Grady, 2007; Cabeza \& Nyberg, 2000; Lepage et al., 2000; Moscovitch et al., 2005; Prince et al., 2005, 2007; Rajah \& McIntosh, 2005; Wiggs et al., 1999). It is hypothesized that episodic encoding is critically dependent upon the integrity of the semantic memory system, because all information encoded into episodic memory first passes through and activates the semantic memory system (Nyberg et al., 1996; Prince et al., 2007; Tulving et al., 1994; Tulving \& Marko-

Correspondence and reprint requests to: David Q. Beversdorf, 300 Portland Street, Suite 110, Columbia, MO 65211. E-mail: beversdorfd@ health.missouri.edu witsch, 1998). Episodic memory tasks instructing subjects to elaborate on the semantic features of objects during learning result in increased encoding (Craik \& Tulving, 1975; Schulman, 1971), presumably by increasing semantic retrieval beyond the baseline level normally invoked in episodic tasks without such instructions. Therefore, it is reasoned that greater activation of the semantic memory system can increase episodic encoding and enhance subsequent episodic retrieval.

Presentation of emotionally negative material during an episodic memory task activates the amygdala (Breiter et al., 1996; Hariri et al., 2002; Morris et al., 1996) and stimulates the central norepinephrine (NE) system (McGaugh et al., 1996; Strange \& Dolan, 2004), which then increases attentional orienting (Anderson \& Phelps, 2001; Öhman et al., 2001) and enhances encoding through interaction with the hippocampal/medial temporal lobe complex (HC/MTL) (Dolcos et al., 2004; Phelps, 2004; Richardson et al., 2004); increasing episodic memory performance for the emotional material (Cahill et al., 1994, 1996; Cahill \& McGaugh, 1995). Accordingly, amygdala lesions (Adolphs et al., 1997; Brierley et al., 2004; Markowitsch et al., 1994) or administration 
of centrally acting $\beta$-adrenergic receptor antagonists (Cahill et al., 1994; van Stegeren et al., 1998) reduce memory enhancements attributable to emotional arousal. Because declarative memory is processed by the HC/MTL at the level of encoding (Cabeza \& Nyberg, 2000; Mayes \& Montaldi, 1999), emotionally arousing material that activate the amygdala should also produce enhancements during encoding for semantic and episodic memories, alike. However, because episodic memory involuntarily elicits retrieval of semantic information (Cabeza \& Nyberg, 2000; Nyberg et al., 1996; Tulving et al., 1994) examining the modulatory influence of emotion on semantic memory is especially challenging.

We propose using a modified oddball task to examine the effects of emotional arousal on semantic memory activation and subsequent contributions of semantic memory to episodic free-recall. Oddball tasks rely upon successful detection of deviant "oddball" stimuli among homogeneous "standard" stimuli to produce an attentional orienting response and enhanced encoding (Strange et al., 2000), which increases subsequent recall of the deviant stimuli; an effect known as the von Restorff phenomenon (for review, see Wallace, 1965). Oddball studies that serially present standard stimuli and randomly insert oddballs have previously been used to demonstrate enhanced episodic free-recall for emotional oddballs, and disrupted consolidation for neutral stimuli preceding an emotional oddball (Hurlemann et al., 2005; Miu et al., 2005; Strange et al., 2003; Tulving, 1969). Increased encoding and successful remembering of the emotionally negative oddball predicts the disrupted consolidation and subsequent forgetting of the preceding neutral stimulus (Strange et al., 2003). Due to the facilitative role of semantic memory in episodic free-recall, and naturalistic accounts of increased memory for the moments preceding traumatic events (Ehlers et al., 2004, 2002), we will examine whether a semantic relationship between an oddball and preceding stimuli can reverse disrupted consolidation and be modulated by negative emotion, resulting in greater episodic free-recall.

To investigate the interaction between semantic relationships and emotion, two structural modifications to the previously described oddball tasks are performed for the present task to study the interactions between emotion and semantic and episodic memory. First, the semantic relationship between the oddball and the immediately preceding word is manipulated, either related or unrelated. Most of the relationships used for this study are associative relationships, such as burn-fire, or lexical co-occurrences, such as cockroach-dirt (Lund \& Burgess, 1996; Moss et al., 1995). Semantic relationships such as these are used extensively in priming studies to show that the presentation of one word facilitates the retrieval of semantically related counterparts (Nelson \& Goodmon, 2002; Tulving \& Schacter, 1990). Next, the emotional impact of the oddball and preceding word is manipulated to include either emotionally negative (E) or neutral (N) words, yielding four conditions; an E word preceding an E oddball, $\mathrm{N}$ preceding an $\mathrm{E}$ oddball, E preceding an $\mathrm{N}$ oddball, and an $\mathrm{N}$ preceding an
$\mathrm{N}$ oddball. Therefore, emotional intensity can be modulated for the presentation of either word (oddball or preceding word), while the relative activation of semantic memory is only modulated upon the presentation of the oddball word, at which time a semantic relationship will or will not be evident.

Using the modified serial-presentation oddball task, we hypothesize that semantically related word pairs will increase semantic memory activation upon the presentation of second word in the associated pair, the oddball, resulting in greater free-recall of both words. Furthermore, we propose that upon presentation of emotionally negative oddballs for related word pairs, semantic memory encoding will be enhanced versus neutral oddballs. Words preceding an emotional oddball will benefit and be recalled significantly better than those preceding neutral oddballs, because increased encoding due to the semantic associations between words will occur in semantic memory. Such an effect will demonstrate the ability of emotion and semantic memory to interact to mutually enhance episodic free-recall.

\section{METHODS}

\section{Participants}

Twenty-eight paid subjects (14 male, 14 female; average age of $22.4 \pm 2.5$ years; average education level of $15.6 \pm$ 1.5 years) were solicited through online advertisements and participated in this study after written informed consent. No participants offered a history of neurological or psychiatric illness or were currently taking medications to treat such illnesses. This protocol was approved by the Institutional Review Board of The Ohio State University.

\section{Stimuli}

Eight words, including exactly 1 oddball word, comprised each of 48 word lists. Within each list, a perceptual oddball word was inserted, in pseudorandom manner, at serial position $4,5,6$, or 7 . The oddball text was lime green in color, while standard stimuli text was white; both presented on a black background with all other perceptual characteristics remaining constant. Oddball words (W), and the words immediately preceding the oddball in each list $(\mathrm{W}-1)$ were chosen from the affective norms for English words (ANEW) (Bradley \& Lang, 1999), with the exception of five words that did not originate from the ANEW list, but were chosen by the experimenter to create meaningful associative relationships, as later described. The remaining six words in each list (288 total) consisted of unique, randomly chosen, general and picturable nouns from Ogden's Basic Word List (Ogden, 1932) and were not strongly associated with W or $\mathrm{W}-1$. The emotionality of the words chosen from the ANEW (positions $\mathrm{W}-1$ and $\mathrm{W}$ ) and their associative relationship to one another yielded 8 different experimental list-type conditions, as demonstrated in Table 1. 
Table 1. W-1 and W ANEW words for each list-type condition

\begin{tabular}{|c|c|c|c|}
\hline \multicolumn{4}{|c|}{ Related } \\
\hline Emotional - Emotional & Neutral - Emotional & Emotional - Neutral & Neutral - Neutral \\
\hline Intruder - Rape & Street - Gang & Ulcer - Stomach & Paper - Pencil \\
\hline Terrorist - Bomb & Lamb - Slaughter & Cockroach - Dirt & Elbow - Arm \\
\hline Snake - Venom & Nun - Devil & Amputate - Foot & Cow - Milk \\
\hline Bloody - Mutilate & Metal - Dagger & Arson - Building & Table - Chair \\
\hline Surgery - Tumor & Cord - Strangle & Gun - Barrel & Key - Door \\
\hline Burn - Fire & Hairdryer - Electrocute & Crash - Bus & Square - Circle \\
\hline \multicolumn{4}{|c|}{ Unrelated } \\
\hline Emotional - Emotional & Neutral - Emotional & Emotional - Neutral & Neutral - Neutral \\
\hline Cancer - Hurricane & Butter - Disaster & Shark - Umbrella & Egg - Curtains \\
\hline Drown - Slave & Basket - Pervert & Crucify - Jelly & Machine - Chin \\
\hline Leprosy - Hostage & Prairie - Killer & Abuse - Banner & Habit - Taxi \\
\hline Bankrupt - Rage & Salad - Assault & Murderer - Bowl & Utensil - Plant \\
\hline Torture - Pollute & Iron - Trauma & Cyclone - Ink & Bench - Horse \\
\hline Nightmare - Thief & Museum - Slap & Ambulance - Farm & Finger - Statue \\
\hline
\end{tabular}

\section{Procedure}

Participants performed the experiment in a well-lit room while seated across from the experimenter. Using E-prime software (Psychological Software Tools, Inc., Pittsburgh, PA) the words were presented on a 15-inch diagonal screen. Instructions and a practice list were administered before beginning the experiment. During the experiment, words in each list were serially presented on a black background for $1 \mathrm{~s}$ each. Between each word, a 1-s rest slide consisting of a white "+" on a black background was presented, resulting in a stimulus onset asynchrony of $2 \mathrm{~s}$. An audible tone indicated the end of a list and the beginning of a 20-s distractor task used to reduce the primacy and recency effects commonly observed in studies of immediate free-recall (Jahnke, 1965). This distractor task required participants to continually subtract 7 from a random three-digit number presented on screen, after which, another audible tone sounded, prompting participants to orally "Please Recall" as many words from the most recently presented list as possible, in any order. On screen instructions also prompted participants to hit the space bar to advance to the next list when finished with free-recall.

The 48 lists of words were presented to each participant in two pseudorandomly ordered blocks of 24 lists, counterbalanced across subject, with an optional 3-min break offered between the blocks. Following presentation of all 48 lists, ratings of arousal and relatedness were taken for each word pair presented in Table 1. During the rating tasks, both words were presented simultaneously on screen, separated by a hyphen, while word order within each pair was consistent with presentation order during the memory task. Arousal for the word pairs was measured using a 9-point version of the arousal portion of the Standard Assessment Manikin (SAM) (Bradley \& Lang, 1994). The SAM nonverbally measures arousal using a pictorially-based scale, therefore reducing cultural language bias. Relatedness for the word pairs was measured using a 9-point scale, where 1 corresponded to "unrelated" and 9 corresponded to "related." This task concluded the experiment and participants were debriefed and released.

\section{Analysis}

\section{Preliminary analyses and analysis design}

SPSS was used for data analysis. To ensure homogeneous distribution of arousing words across list-type conditions, subjective arousal ratings from Bradley \& Lang (1999) for ANEW words (positions $\mathrm{W}-1$ and $\mathrm{W}$ ) were compared using independent sample $t$ tests before beginning the study. Emotion - emotion (EE) and neutral - neutral (NN) word pairs were compared across the relatedness condition, while emotion - neutral (EN) and neutral - emotion (NE) word pairs were compared with each other within and across the relatedness condition.

A one-way ANOVA examining overall memory performance across each list-type condition was performed. Any significant difference in free-recall performance across listtype conditions would require that free-recall scores be adjusted and reported as a percentage of control word (C) performance for each condition. Control word performance for each participant was established by assessing delayed freerecall memory on a random word from any position in each list, excluding positions $\mathrm{W}-1, \mathrm{~W}$, or $\mathrm{W}+1$ (48 instances per participant; 1344 total instances). Using these difference scores, a $4 \times 2 \times 2 \times 2$ repeated-measures ANOVA (position $\times$ associative relatedness $\times$ emotionality of $\mathrm{W} \times$ emotionality of $\mathrm{W}-1$ ) was performed to examine delayed freerecall memory performance. The first variable, position, considered the serial position of words relative to the oddball, including control word performance; $\mathrm{W}-1, \mathrm{~W}, \mathrm{~W}+1$, 
and $\mathrm{C}$. The second variable considered the semantic relationship between $\mathrm{W}$ and $\mathrm{W}-1$; either semantically related or unrelated. The final two variables considered the emotionality of $\mathrm{W}$ and $\mathrm{W}-1$; either emotionally negative or neutral.

To examine relative enhancements due specifically to semantic relatedness, unrelated free-recall performance was subtracted from related free-recall performance for each of the EE, NE, EN, and NN conditions within each serial position. It was assumed that semantic contributions to episodic free-recall could be obtained by subtracting performance in the unrelated condition from performance in the related condition. Because the subtraction left only enhancements due to semantic memory contributions and we are only interested in a single serial position for these analyses, a $2 \times 2$ ANOVA resulted from the remaining factors (emotionality of $\mathrm{W} \times$ emotionality of $\mathrm{W}-1$ ). Where applicable, significant main and interaction effects were further analyzed using Bonferroni-corrected independent-sample $t$ tests and corrected $p$ values are reported.

\section{Emotional modulation of semantic memory and contributions to episodic free recall}

Using the $4 \times 2 \times 2 \times 2$ repeated-measures ANOVA described above, we examined semantic memory contributions to episodic free-recall. A significant main effect for relatedness and significant interaction effects for position $\times$ relatedness, specifically increased free-recall memory performance for associatively related words in both the $\mathrm{W}-1$ and $\mathrm{W}$ positions would verify our hypothesis that semantic memory activation significantly contributes to episodic free-recall.

A significant interaction effect for position $\times$ relatedness $\times \mathrm{W}$ emotion using the $4 \times 2 \times 2 \times 2$ repeatedmeasures ANOVA described above would be consistent with our hypothesis that emotionally negative words can modulate semantic memory during encoding, and that emotional modulation of semantic memory can contribute to episodic free-recall. However, subsequent analyses examining this significant interaction for serial positions $\mathrm{W}$ and $\mathrm{W}-1$ using the above described $2 \times 2$ ANOVA would be necessary to confirm each hypothesis. For stimuli in the W position, increased free-recall for emotional oddballs, as indicated by a significant main effect for $\mathrm{W}$ emotionality, would confirm our hypothesis that semantic memory is modulated by emotion, similar to episodic memory. For related stimuli in the $\mathrm{W}-1$ position, increased recall of words preceding negative oddballs, as indicated by a significant main effect for W emotionality, would indicate that increased semantic memory encoding due to emotionally negative stimuli contributes to episodic free-recall.

\section{Subjective ratings of arousal and relatedness}

Subjective ratings of arousal and relatedness were analyzed using a $2 \times 2 \times 2$ repeated-measures ANOVA (relatedness $\times$ emotionality of $\mathrm{W} \times$ emotionality of $\mathrm{W}-1$ ). Signif- icant main effects for the emotionality of $\mathrm{W}$ or $\mathrm{W}-1$ showing greater ratings of arousal would indicate higher subjective arousal for the arousing stimuli. A significant main effect for relatedness would indicate that participants reported greater amounts of relatedness for related stimuli.

\section{RESULTS}

\section{Preliminary Analyses}

An a priori analysis of subjective arousal ratings from Bradley \& Lang (1999) found no significant differences across the compared experimental conditions (related $v s$. unrelated and EN vs. NE), thus ensuring homogeneous distribution of arousing words. Overall free-recall performance significantly differed across each list-type condition, $F(1,7)=2.08 ; p<.05$, which required free-recall scores to be adjusted and reported as a proportion of control word performance. No primacy or recency effects were observed for control words.

\section{Emotional Modulation of Semantic Memory and Contributions to Episodic Free Recall}

The $4 \times 2 \times 2 \times 2$ repeated-measures ANOVA examining overall memory performance revealed a significant main effect for serial word position relative to the oddball, $F(3,81)=$ 104.17; $p<.001$, as shown in Figure 1. Further analysis revealed that oddball words $(\mathrm{W})$ were remembered significantly better than control words $(\mathrm{C}), t(446)=12.64 ; p<.001$, verifying our ability to reproduce the von Restorff effect. A significant decrease in free-recall is observed for words following the oddball, $t(446)=-3.77 ; p=.006$. A significant main effect was also found for relatedness, $F(1,27)=57.74$; $p<.001$, which indicated that semantic activation significantly contributed to episodic free-recall. Bonferronicorrected $t$ tests examining related versus unrelated stimuli in serial positions $\mathrm{W}-1$ and $\mathrm{W}$ revealed significantly increased free-recall for related stimuli, $t(110)=3.086 ; p=.041$ for $\mathrm{W}-1$ and $t(110)=3.189 ; p=.030$ for $\mathrm{W}$.

Consistent with our hypothesis that emotionally negative words can enhance semantic contributions to episodic freerecall during encoding, a significant position $\times$ associative relatedness $\times \mathrm{W}$ emotionality interaction effect was revealed by the $4 \times 2 \times 2 \times 2$ repeated-measures ANOVA, $F(3,81)=$ $3.16 ; p=.029$. Further examination of stimuli in the $\mathrm{W}$ position, using the above described $2 \times 2$ ANOVA for freerecall enhancements due to semantic contributions, revealed a significant main effect of $\mathrm{W}$ emotionality, $F(1,27)=5.94$; $p=.022$, confirming our hypothesis that emotionally negative material enhanced semantic contributions to episodic memory, as seen in Figure 2. Using the $2 \times 2$ ANOVA examining enhancements due to semantic memory, a significant main effect of $\mathrm{W}$ emotionality was also observed upon for stimuli in the $\mathrm{W}-1$ position, $F(1,27)=13.54 ; p=$ .001 . This result, portrayed in Figure 3, confirmed our hypothesis that emotional modulation of semantic memory 


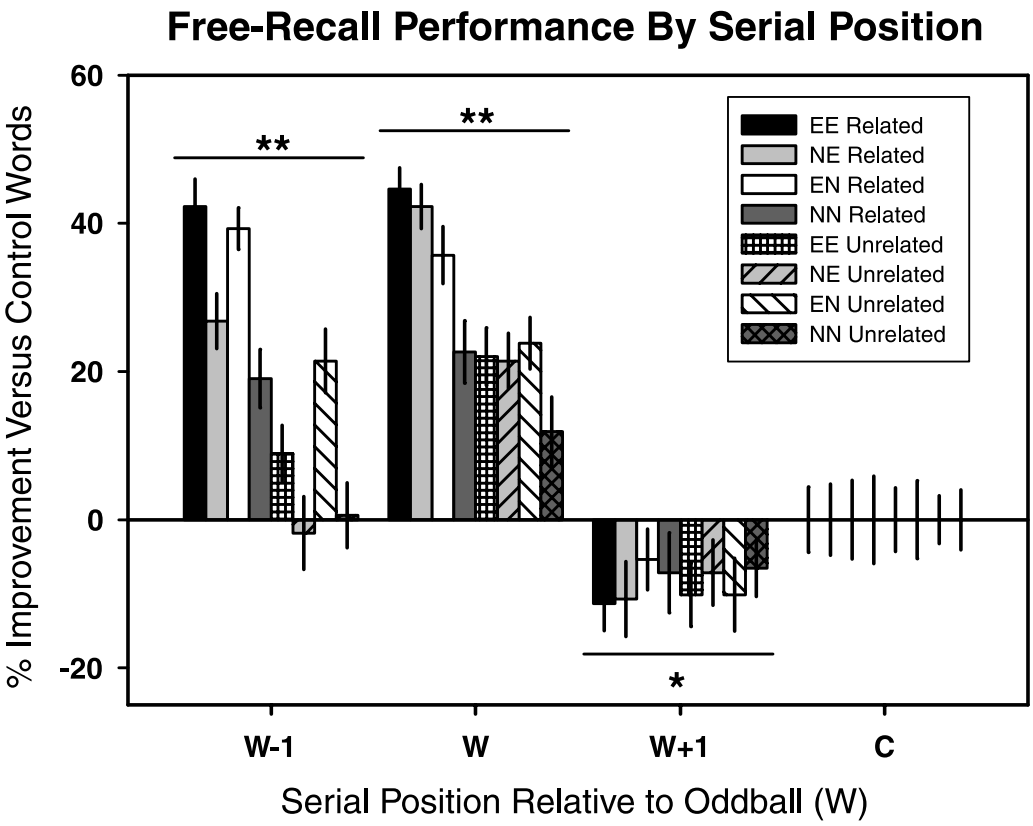

Fig. 1. Overall memory performance for $\mathrm{W}-1, \mathrm{~W}$, and $\mathrm{W}+1$ relative to control word performance. Memory performance for all positions differed significantly from control words. Oddball words (W) were remembered significantly better than controls words (C), replicating the von Restorff effect. Words preceding the oddball $(\mathrm{W}-1)$ were also remembered significantly better overall than control words. Words following the oddball $(\mathrm{W}+1)$ were remembered significantly less than control words. ${ }^{*} p<.01$ $* * p<.001$. encoding significantly contributed to the episodic freerecall of $\mathrm{W}-1$ stimuli.

\section{Subjective Ratings of Arousal and Relatedness}

Word pairs containing one or more emotionally arousing word were rated significantly more arousing, regardless of

\section{Semantic Contributions to Oddball Free-Recall}

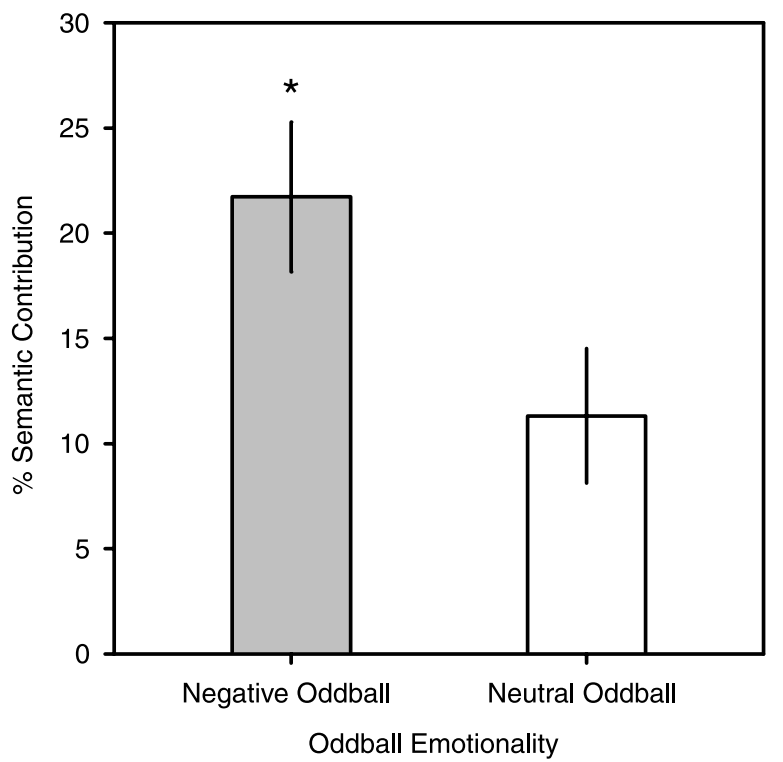

Fig. 2. Memory enhancements for emotional versus neutral oddball stimuli. Semantic contributions to free-recall memory was significantly higher for emotional versus neutral oddball stimuli. $* p<.05$. whether the emotional word appeared in the $\mathrm{W}$ or $\mathrm{W}-1$ position, $F(1,27)=62.50 ; p<.001$ and $F(1,27)=55.19$; $p<.001$, respectively. Analysis of subjective relatedness ratings revealed that related word pairs were rated significantly more related than unrelated pairs, $F(1,27)=1175.50$; $p<.001$. Analysis of subjective arousal ratings revealed that related word pairs were rated significantly more arousing by participants, $F(1,27)=17.69 ; p<.001$, despite no significant differences across lists on preliminary analyses of subjective arousal using data from Bradley \& Lang (1999). Likewise, the appearance of an emotional word in the $\mathrm{W}$ or $\mathrm{W}-1$ position resulted in significantly greater ratings of

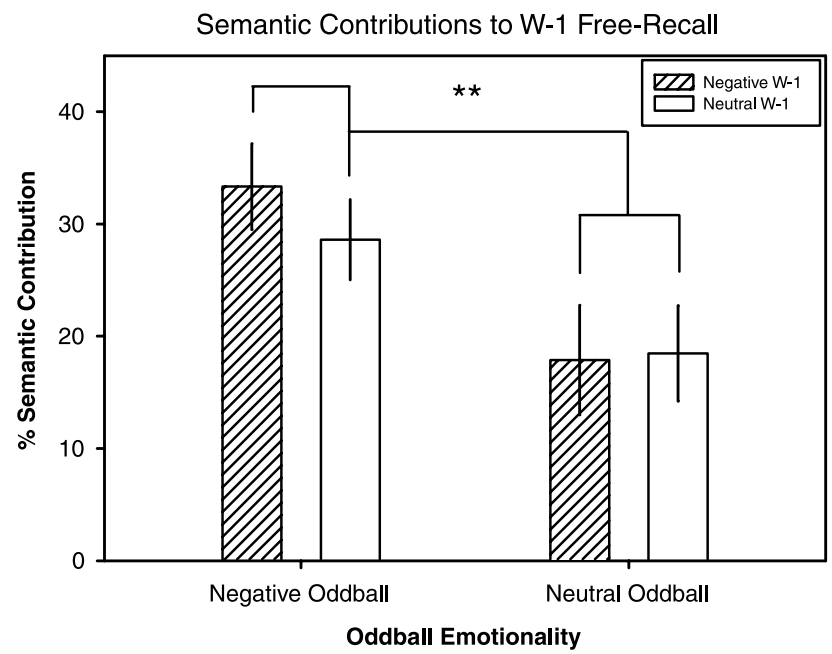

Fig. 3. Semantic contributions to free-recall for related $W-1$ words, grouped by oddball (W) emotionality and $\mathrm{W}-1$ emotionality. Semantic contributions to free-recall for related words in the $\mathrm{W}-1$ position were significantly greater when preceding an emotionally negative oddball word. $* * p=.001$. 
relatedness, $F(1,27)=25.81 ; p<.001$ and $F(1,27)=47.50$; $p<.001$, respectively. This finding was likely driven by the significantly increased subjective relatedness ratings for the emotional $\mathrm{W} \times$ emotional $\mathrm{W}-1$ condition versus all other unrelated conditions, $p<.001$ for all pairwise comparisons. Figures $4 \mathrm{a}$ and $4 \mathrm{~b}$ summarize obtained subjective rating data.

\section{DISCUSSION}

The present study demonstrates that semantic memory and negative emotion can interact to mutually increase episodic free-recall. It has long been known that activation of semantic memory during episodic encoding can greatly increase later episodic free-recall or recognition (Craik \& Tulving,

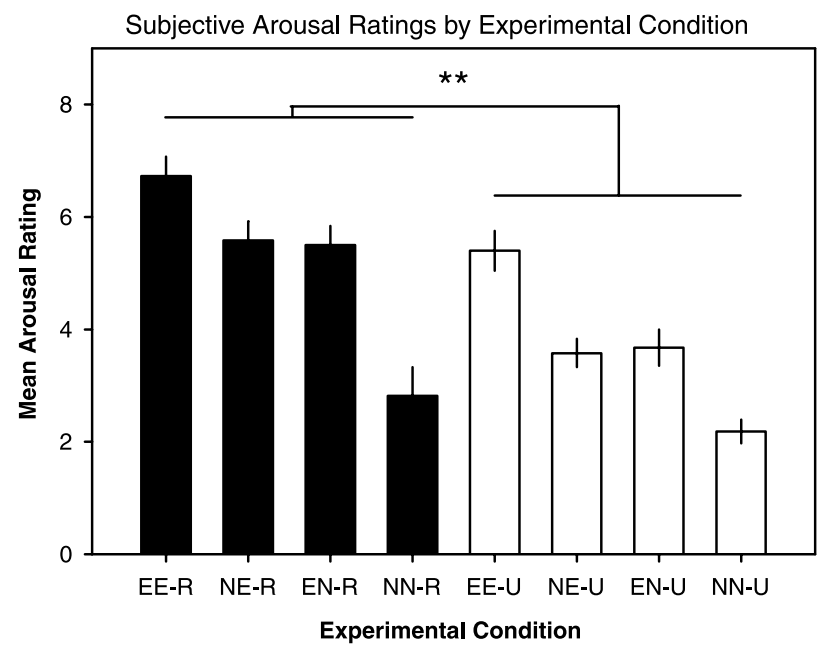

Subjective Relatedness Ratings by Experimental Condition

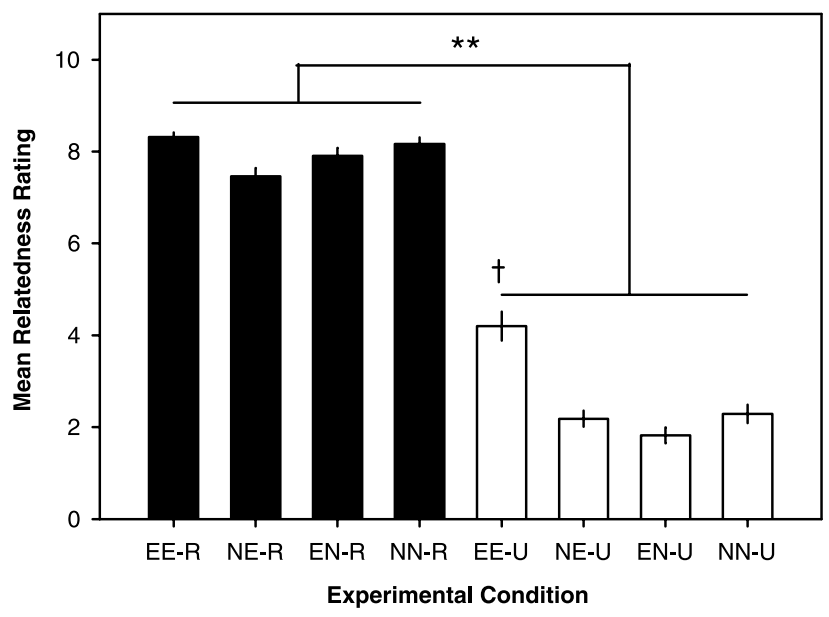

Fig. 4. a,b: Note. $\mathrm{E}=$ emotionally negative, $\mathrm{N}=$ neutral, $\mathrm{R}=$ related, and $\mathrm{U}=$ unrelated. Mean subjective arousal (a) and relatedness (b) ratings for each experimental condition. Subjective ratings of arousal and relatedness are significantly increased for related stimuli versus unrelated stimuli and for stimuli in the emotionally negative versus neutral category. ${ }^{* *} p<.001$ and $\dagger p<.001$ versus all other unrelated list-type conditions.
1975; Schulman, 1971). However, the use of a serial presentation design during the present study allows for the demonstration of what appears to be a retroactive episodic memory increase due to contributions by the semantic memory system interacting with negative emotion. That is, freerecall enhancements for the earlier presented word are dependent upon the presentation of a second semantically related word which is further modulated by negative emotion. Negative emotion can also decrease free-recall for words following oddballs, as seen in Figure 1, similar to the reductions observed in previous studies (Hurlemann et al., 2005; Miu et al., 2005).

Two possible factors could significantly contribute to a retroactive memory increase for semantically related words. Presentation of the second word might bolster the consolidation of the earlier presented word within episodic memory. Alternatively, presentation of the second related word results in greater semantic memory activation, creating stronger semantic memory cues that are available upon elicitation of episodic free-recall. Findings from the present study favor the latter explanation: previous oddball tasks demonstrate that items immediately preceding emotional oddballs are subject to consolidation disruptions (Hurlemann et al., 2005; Miu et al., 2005; Strange et al., 2003; Tulving, 1969). However, we find that words preceding and semantically related to negative oddballs are actually recalled better than those preceding and related to neutral oddballs, while both are recalled significantly better than words not semantically related to the oddball. It seems less likely that a bi-directional modulation for consolidation occurs based solely on the presence of a semantic relationship, especially because the presence of a semantic relationship does not manifest until the presentation of the second word. Instead, we find it more likely that when episodic encoding activates the retrieval of semantic information (Nyberg et al., 1996; Prince et al., 2007; Tulving et al., 1994; Tulving \& Markowitsch, 1998), it does so to varying degrees, based on the semantic associativity between the two items. Upon episodic memory retrieval, the semantic network is concurrently activated, and similar to the spreading-activation theory of semantic processing (Collins \& Loftus, 1975), recall of any given word facilitates the recall of a second word to a degree proportional to the semantic relatedness between the two words. In summary, semantic memory activation occurs concurrently with episodic encoding and varies in intensity as a function of the relatedness between any two items within the semantic network on verbal tasks such as ours.

Negative emotion appears to modulate semantic memory in a manner consistent with that seen for episodic memory contributions. If emotionally negative material simply increases episodic encoding, it is expected that related words preceding the oddballs are not influenced by the emotional impact of the later presented oddballs. In the present study, however, increased episodic free-recall is observed for both emotionally negative oddballs and related words preceding the emotionally negative oddball, as compared to those 
related words preceding neutral oddballs (Figures 3 and 4). According to subjective ratings of arousal collected for this study, it also appears that semantic relationships can significantly increase arousal ratings (Figure 4a), perhaps further contributing to episodic free-recall.

The finding by Strange et al. (2003), demonstrating that the recall of the emotional word inhibited rather than facilitated the recall of the previous neutral word, appear to be in stark contrast to the above conclusion. However, the control words used by Strange et al. (2003) all shared a semantic category, while the emotional oddball words shared only a weak semantic relationship to the preceding neutral words and had no incongruent perceptual characteristic. This differs significantly from the present study in which control words were purposely not semantically related, while the emotional oddball and preceding word could share strong semantic ties. Additionally, all oddballs were perceptually incongruent from the standard stimuli, as they were lime green in color, while the emotional oddballs also differed in their emotionality. According to studies of proactive inhibition, continued presentation of words within a single semantic category results in a steady decline in free-recall memory until that proactive inhibition is released by changing a semantic component (Wickens, 1970). Therefore, in the study by Strange et al. (2003), the change in emotional impact upon the presentation of the weakly associated emotionally negative word could initiate a release from proactive inhibition, increasing free-recall for the emotional word, while preceding words show a free-recall decline. As discussed below, varying latencies between stimuli in the study by Strange et al. (2003) and the present study may also account for the observed differences.

Recall for serially presented information is also significantly influenced by the latency of the onset between serially presented stimuli, or SOA. For serial-presentation oddball studies, it appears as if a shorter SOA increases memory impairments for words preceding the oddball (Tulving, 1969). With the exception of the study by Tulving (1969), each of the previously mentioned studies that found memory decrements attributable to the oddball used an SOA that was markedly longer than the $2 \mathrm{~s}$ SOA used in the present study, ranging from 3 s (Miu et al., 2005; Strange et al., 2003) to $5 \mathrm{~s}$ (Hurlemann et al., 2005). The shorter 2-s latency in the present study would, therefore, suggest that stimuli preceding the oddball are within a range susceptible to memory disruptions attributable to the oddball, but the presence of semantic relationships are able to mitigate those disruptions. Additionally, the SOA in the present study is sufficiently longer than the $550 \mathrm{~ms}$ required for the recognition of associative relationships and later declarative memory enhancements (Fischler \& Goodman, 1978).

It is demonstrated through the use of neuroimaging techniques that semantic memory is intimately involved in the encoding and retrieval of episodic memories (Nyberg et al., 1996; Prince et al., 2007; Tulving et al., 1994; Tulving \& Markowitsch, 1998). Therefore, understanding the modulatory role of emotion on semantic memory can contribute to the understanding of episodic memory formation and retrieval. Interactions between emotion and memory comprise fundamental components of several psychological disorders, such as intrusive memory symptoms in posttraumatic stress disorder (PTSD) (Ehlers et al., 2002, 2004). Using the proposed oddball task on such populations might be beneficial in elucidating fundamental disturbances in interactions between emotion and semantic or episodic memory. Future work also needs to consider the temporal characteristics of the enhancements observed here. Changes in the SOA or time between learning and retrieval are likely to further clarify the roles of encoding versus consolidation. Institution of "know" versus "remember" designations (Tulving, 1985; Yonelinas, 2002) for delayed recognition memory using this task could also help determine the relative contribution of semantic activation to episodic memory. An overall understanding of naturalistic memory formation will require a better appreciation of contributions and interactions between memory systems studied in the laboratory.

\section{ACKNOWLEDGMENTS}

This research was funded in part by grants from NIDA (R21 DA015734) (Beversdorf) and NINDS (K23 NS43222) (Beversdorf). No financial or other relationships currently exist that could be interpreted as a conflict of interest affecting this manuscript. Portions of this research were presented at the Cognitive Neuroscience Society, 2007.

\section{REFERENCES}

Adolphs, R., Cahill, L., Schul, R., \& Babinsky, R. (1997). Impaired declarative memory for emotional material following bilateral amygdala damage in humans. Learning \& Memory, 4, 291-300.

Anderson, A.K. \& Phelps, E.A. (2001). Lesions of the human amygdala impair enhanced perception of emotionally salient events. Nature, 411, 305-309.

Bradley, M.M. \& Lang, P.J. (1994). Measuring emotion: The SelfAssessment Manikin and the Semantic Differential. Journal of Behavior Therapy and Experimental Psychiatry, 25, 49-59.

Bradley, M.M. \& Lang, P.J. (1999). Affective norms for English words (ANEW): Stimuli, instruction manual and affective ratings. Technical report $C$-1. Gainesville, FL: The Center for Research in Psychophysiology, University of Florida.

Breiter, H.C., Etcoff, N.L., Whalen, P.J., Kennedy, W.A., Rauch, S.L., Buckner, R.L., Strauss, M.M., Hyman, S.E., \& Rosen, B.R. (1996). Response and habituation of the human amygdala during visual processing of facial expression. Neuron, 17, 875-887.

Brierley, B., Medford, N., Shaw, P., \& David, A.S. (2004). Emotional memory and perception in temporal lobectomy patients with amygdala damage. Journal of Neurology, Neurosurgery, and Psychiatry, 75, 593-599.

Burianova, H. \& Grady, C.L. (2007). Common and unique neural activations in autobiographical, episodic, and semantic retrieval. Journal of Cognitive Neuroscience, 19, 1520-1534.

Cabeza, R. \& Nyberg, L. (2000). Imaging cognition II: An empirical review of 275 PET and fMRI studies. Journal of Cognitive Neuroscience, 12, 1-47. 
Cahill, L., Haier, R.J., Fallon, J., Alkire, M.T., Tang, C., Keator, D., Wu, J., \& McGaugh, J.L. (1996). Amygdala activity at encoding correlated with long-term, free recall of emotional information. Proceedings of the National Academy of Sciences of the United States of America, 93, 8016-8021.

Cahill, L. \& McGaugh, J.L. (1995). A novel demonstration of enhanced memory associated with emotional arousal. Consciousness and Cognition, 4, 410-421.

Cahill, L., Prins, B., Weber, M., \& McGaugh, J.L. (1994). $\beta$-Adrenergic activation and memory for emotional events. Nature, 371, 702-704.

Collins, A.M. \& Loftus, E.F. (1975). A spreading-activation theory of semantic processing. Psychological Review, 82, 407-428.

Craik, F.I.M. \& Tulving, E. (1975). Depth of processing and the retention of words in episodic memory. Journal of Experimental Psychology: General, 104, 268-294.

Dolcos, F., LaBar, K.S., \& Cabeza, R. (2004). Interaction between the amygdala and the medial temporal lobe memory system predicts better memory for emotional events. Neuron, 42, $855-863$.

Ehlers, A., Hackmann, A., \& Michael, T. (2004). Intrusive re-experiencing in post-traumatic stress disorder: Phenomenology, theory, and therapy. Memory, 12, 403-415.

Ehlers, A., Hackmann, A., Steil, R., Clohessy, S., Wenninger, K., \& Winter, H. (2002). The nature of intrusive memories after trauma: The warning signal hypothesis. Behaviour Research and Therapy, 40, 995-1002.

Fischler, I. \& Goodman, G.O. (1978). Latency of associative activation in memory. Journal of Experimental Psychology: Human Perception and Performance, 4, 455-470.

Hariri, A.R., Tessitore, A., Mattay, V.S., Fera, F., \& Weinberger, D.R. (2002). The amygdala response to emotional stimuli: A comparison to faces and scenes. Neuroimage, 17, 317-323.

Hurlemann, R., Hawellek, B., Matusch, A., Kolsch, H., Wollersen, H., Madea, B., Vogeley, K., Maier, W., \& Dolan, R.J. (2005). Noradrenergic modulation of emotion-induced forgetting and remembering. The Journal of Neuroscience, 25, 6343-6349.

Jahnke, J.C. (1965). Primacy and recency effects in serial-position curves of immediate recall. Journal of Experimental Psychology, 70, 130-132.

Lepage, M., Habib, R., Cormier, H., Houle, S., \& McIntosh, A.R. (2000). Neural correlates of semantic associative encoding in episodic memory. Cognitive Brain Research, 9, 271-280.

Lund, K. \& Burgess, C. (1996). Producing high-dimensional semantic spaces from lexical co-occurrence. Behavior Research Methods, Instruments, \& Computers, 28, 203-208.

Markowitsch, H.J., Calabrese, P., Würker, M., Durwen, H.F., Kessler, J., Babinsky, R., Brechtelsbauer, D., Heuser, L., \& Gehlen, W. (1994). The amygdala's contribution to memory-a study on two patients with Ubach-Wiethe disease. Neuroreport, 5, 1349-1352.

Mayes, A.R. \& Montaldi, D. (1999). The neuroimaging of longerterm memory encoding processes. Memory, 7, 613-659.

McGaugh, J.L., Cahill, L., \& Roozendaal, B. (1996). Involvement of the amygdala in memory storage: Interaction with other brain systems. Proceedings of the National Academy of Sciences of the United States of America, 93, 13508-13514.

Miu, A.C., Heilman, R.M., Opre, A., \& Miclea, M. (2005). Emotioninduced retrograde amnesia and trait anxiety. Journal of Experimental Psychology: Learning, Memory, and Cognition, 31, 1250-1257.
Morris, J.S., Frith, C.D., Perrett, D.I., Rowland, D., Young, A.W., Calder, A.J., \& Dolan, R.J. (1996). A differential neural response in the human amygdala to fearful and happy facial expressions. Nature, 383, 812-815.

Moscovitch, M., Rosenbaum, R.S., Gilboa, A., Addis, D.R., Westmacott, R., Grady, C., McAndrews, M.P., Levine, B., Black, S., Winocur, G., \& Nadel, L. (2005). Functional neuroanatomy of remote episodic, semantic, and spatial memory: A unifies account based on multiple trace theory. Journal of Anatomy, 207, 35-66.

Moss, H.E., Ostrin, R.K., Tyler, L.K., \& Marslen-Wilson, W.D. (1995). Accessing different types of lexical semantic information: Evidence from priming. Journal of Experimental Psychology: Learning, Memory, and Cognition, 21, 863-883.

Nelson, D.L. \& Goodmon, L.B. (2002). Experiencing a word can prime its accessibility and its associative connections to related words. Memory \& Cognition, 30, 380-398.

Nyberg, L., Cabeza, R., \& Tulving, E. (1996). PET studies of encoding and retrieval: The HERA model. Psychonomic Bulletin \& Review, 3, 135-148.

Ogden, C.K. (1932). Basic English: A general introduction with rules and grammar (3rd ed.). London: K. Paul, Trench, Trubner \& Co., Ltd.

Öhman, A., Flykt, A., \& Esteves, F. (2001). Emotion drives attention: Detecting the snake in the grass. Journal of Experimental Psychology: General, 130, 466-478.

Phelps, E.A. (2004). Human emotion and memory: Interaction of the amygdala and hippocampal complex. Current Opinion in Neurobiology, 14, 198-202.

Prince, S.E., Daselaar, S.M., \& Cabeza, R. (2005). Neural correlates of relational memory: Successful encoding and retrieval of semantic and perceptual associations. The Journal of Neuroscience, 25, 1203-1210.

Prince, S.E., Tsukiura, T., \& Cabeza, R. (2007). Distinguishing the neural correlates of episodic memory encoding and semantic memory retrieval. Psychological Science, 18, 144-151.

Rajah, M.N. \& McIntosh, A.R. (2005). Overlap in the functional neural systems involves in semantic and episodic memory retrieval. Journal of Cognitive Neuroscience, 17, 470-482.

Richardson, M.P., Strange, B.A., \& Dolan, R.J. (2004). Encoding of emotional memories depends on amygdala and hippocampus and their interactions. Nature Neuroscience, 7, 278-285.

Schulman, A.I. (1971). Recognition memory for targets from a scanned word list. British Journal of Psychology, 62, 335-346.

Strange, B.A. \& Dolan, R.J. (2004). $\beta$-Adrenergic modulation of emotional memory-evoked human amygdala and hippocampal responses. Proceedings of the National Academy of Sciences of the United States of America, 101, 11454-11458.

Strange, B.A., Henson, R.N.A., Friston, K.J., \& Dolan, R.J. (2000). Brain mechanisms for detecting perceptual, semantic, and emotional deviance. Neuroimage, 12, 425-433.

Strange, B.A., Hurlemann, R., \& Dolan, R.J. (2003). An emotioninduced retrograde amnesia in humans is amygdala- and $\beta$-adrenergic-dependent. Proceedings of the National Academy of Sciences of the United States of America, 100, 1362613631.

Tulving, E. (1969). Retrograde amnesia in free recall. Science, 164, 88-90.

Tulving, E. (1983). Elements of episodic memory. New York: Oxford University Press.

Tulving, E. (1985). Memory and consciousness. Canadian Psychology, 26, 1-12. 
Tulving, E. (2002). Episodic memory: From mind to brain. Annual Review of Psychology, 53, 1-25.

Tulving, E., Kapur, S., Craik, F.I.M., Moscovitch, M., \& Houle, S. (1994). Hemispheric encoding/retrieval asymmetry in episodic memory: Positron emission tomography findings. Proceedings of the National Academy of Sciences of the United States of America, 91, 2016-2020.

Tulving, E. \& Markowitsch, H.J. (1998). Episodic and declarative memory: Role of the hippocampus. Hippocampus, 8, 198-204.

Tulving, E. \& Schacter, D.L. (1990). Priming and human memory systems. Science, 247, 301-306.

van Stegeren, A.H., Everaerd, W., Cahill, L., McGaugh, J.L., \& Gooren, L.J.G. (1998). Memory for emotional events: Differ- ential effects of centrally versus peripherally acting $\beta$-blocking agents. Psychopharmacology, 138, 305-310.

Wallace, W.P. (1965). Review of the historical, empirical, and theoretical status of the von Restorff phenomenon. Psychological Bulletin, 63, 410-424.

Wickens, D.D. (1970). Encoding categories of words: An empirical approach to meaning. Psychological Review, 77, 1-15.

Wiggs, C.L., Weisberg, J., \& Martin, A. (1999). Neural correlates of semantic and episodic memory retrieval. Neuropsychologia, 37, 103-118.

Yonelinas, A.P. (2002). The nature of recollection and familiarity: A review of 30 years of research. Journal of Memory and Language, 46, 441-517. 